\title{
Competition between tunnel- and viscosity-effects on bimolecular hydrogen-transfer reaction
}

\author{
Uwe Kensy, Karl-Heinz Grellmann* and Manuel Mosquera González ${ }^{1)}$ \\ Max-Planck-Institut für Biophysikalische Chemie, D-3400 Göttingen, Germany \\ 1) Permanent address: Universidad de Santiago, Santiago de Compostela, Spain
}

\begin{abstract}
The photocyclization of a methylsubstituted diphenylamine results in the formation of a transient zwitterionic dihydrocarbazole. The transient converts into a stable dihydrocarbazole by an intramolecular, sigmatropic hydrogen shift and by an intermolecular hydrogen exchange reaction. The rates of both reactions are governed by hydrogen tunnel effects. The intermolecular hydrogen exchange is the first example for a bimolecular reaction where tunnel effects have been observed. The role of solvent viscosity is discussed.
\end{abstract}

\section{INTRODUCTION}

In recent years a number of intra molecular hydrogen transfer reactions in the singlet ground state as well as in excited triplet states have been investigated where quantum mechanical tunnel

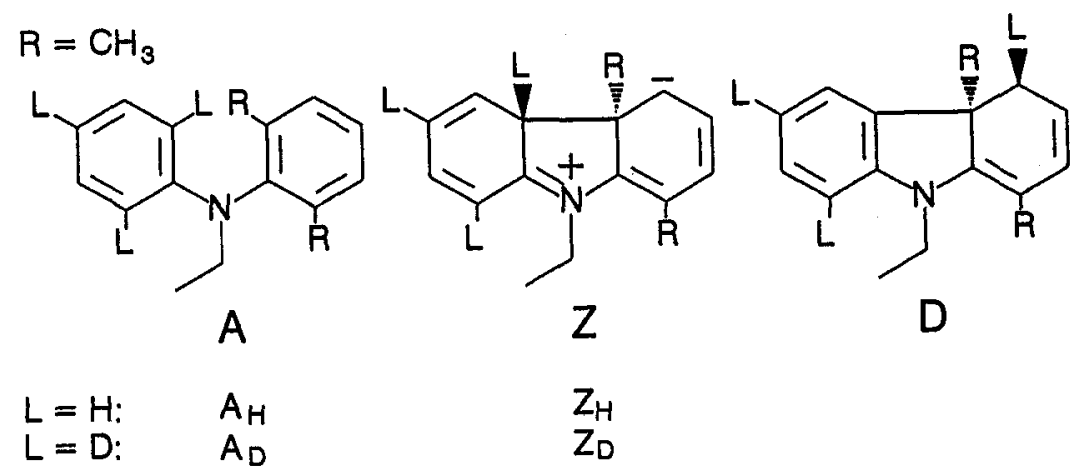

effects clearly determine the rates of the transfer reaction (ref. 1). To our knowledge, no such tunnel effects have been observed so far on an intermolecular hydrogen transfer reaction. In this paper we wish to describe a photoreaction which initiates an intramolecular hydrogen transfer as well as an intermolecular hydrogen exchange reaction.

\section{RESULTS AND DISCUSSION}

The diphenylamine derivative A photocyclizes adiabatically in its triplet state (ref. 2 ), ${ }^{3} \mathrm{~A}^{*}$, yielding the $z$ witterionic intermediate ${ }^{3} Z^{*}$ which subsequently relaxes to its singlet ground state ${ }^{1} Z$. Depending on temperature, the photocyclization ${ }^{3} \mathrm{~A}^{*} \rightarrow{ }^{3} \mathrm{Z}^{*}$ takes place within $\sim 10 \mathrm{~ns}$ at $300 \mathrm{~K}$ 
and $\sim 100 \mu$ s at $100 \mathrm{~K}$; the intersystem crossing process ${ }^{3} Z^{*} \rightarrow{ }^{1} \mathrm{Z}$ is temperature independent (rate constant $\mathrm{k}_{\text {isc }}=3 \times 10^{6} \mathrm{~s}^{-1}$ ). The lifetime of ${ }^{1} \mathrm{Z}$ is at all temperatures several orders of magnitude longer than that of its precursors. Therefore, if one analyses the decay of $1 Z$, which has an absorption maximum at $\lambda_{\max }=520 \mathrm{~nm}$, its formation can be taken to be instantaneous.

Three reaction channels are responsible for the decay of ${ }^{1} Z$ (cf. Scheme 1): 1 . Thermally activated ring-opening back to the starting material (rate constant $k_{b}$ ). 2. Rearrangement by an intramolecular sigmatropic $[1,8]$-hydrogen-shift (rate constant $k_{h s}$ ) yielding as final product the stable dihydrocarbazole D. 3. Intermolecular hydrogen exchange (overall rate constant $\theta_{\text {ex }}$ ) yielding the same endproduct $\mathrm{D}$ as the intramolecular process $\mathrm{k}_{\mathrm{hs}}$.

Scheme 1

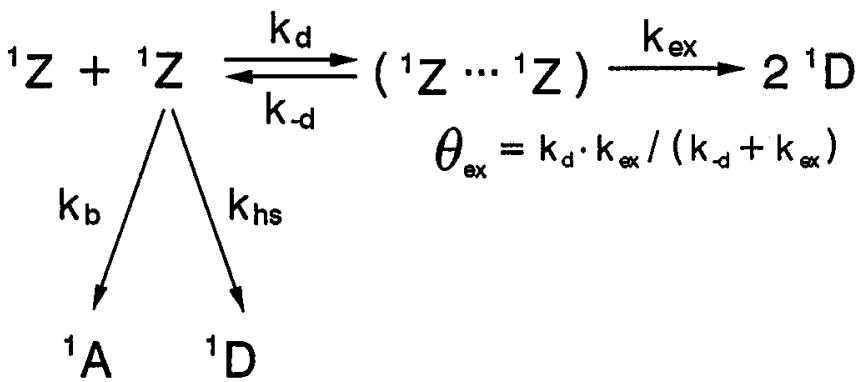

The ring-opening reaction has a high activation energy $\left(k_{b}=5 \times 10^{12} \exp \left(-\Delta \mathrm{E}_{\mathrm{b}} / \mathrm{RT}\right) ; \Delta \mathrm{E}_{\mathrm{b}}=\right.$ $57 \mathrm{~kJ} / \mathrm{mol}$ ). It prevails at temperatures above $\sim 200 \mathrm{~K}$ and therefore, the decay of $1 \mathrm{Z}$ is within experimental error monoexponential in that temperature region. At temperatures below $\sim 180 \mathrm{~K}$ the rates of the intramolecular rearrangement $\left(k_{h s}\right)$ and the bimolecular exchange reaction $\left(\theta_{e x}\right)$ dominate and the decay of ${ }^{1} \mathbf{Z}$ is of mixed first- and second-order.

At $T=173 \mathrm{~K}$, solutions of the amines $A_{H}$ and $A_{D}$ in nitrogen-purged 3-methylpentane (3MP) were photolysed in mg-amounts (ref. 3 ). The resulting photoproducts were carefully analysed by NMR-spectroscopy. According to this analysis only one photoproduct (detection limit $\geq 5 \%$ ) was formed, namely the dihydrocarbazole $D$. After the photolysis of the deuterated compound $A_{D}$ one $\mathrm{D}$-atom was found on the diene-ring of $\mathrm{D}$, the other 2 remained at the aromatic ring. Since at $173 \mathrm{~K}$ both, the first- and the second-order reaction contribute substantially to the decay of ${ }^{1} \mathrm{Z}$, we conclude that the two decay processes yield the same photoproduct $D$.

\section{Scheme 2}
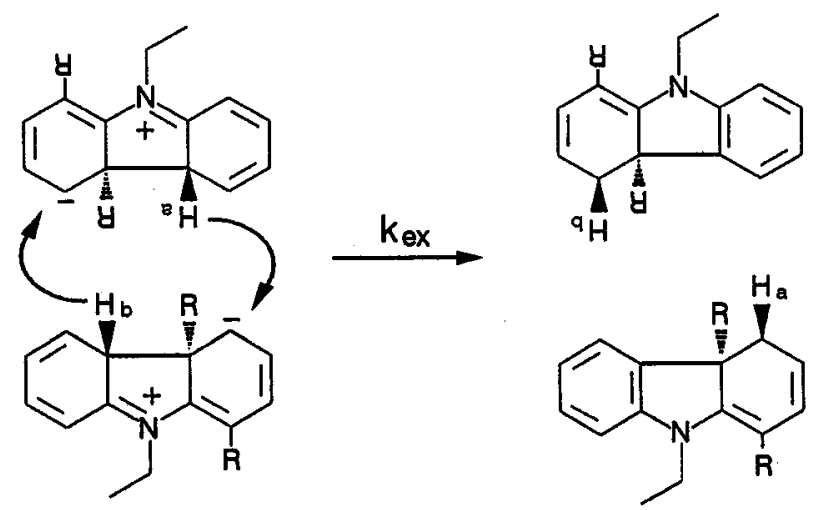
In this paper we discuss the second-order reaction in more detail. We propose that the zwitterion ${ }^{1} Z$ forms an encounter complex $\left({ }^{1} Z \cdots 1 Z\right.$ ) (rate constant $k_{d}$ ) which either dissociates $\left(k_{-d}\right)$ or forms two molecules of ${ }^{1} \mathrm{D}$ by a mutual hydrogen exchange (rate constant $\mathrm{k}_{\mathrm{ex}}$, cf. Scheme 2 ). The overall second-order rate constant of this reaction is

$$
\theta_{e x}=k_{d} \cdot k_{e x} /\left(k_{e x}+k_{-d}\right)
$$

If $\mathrm{k}_{\mathrm{ex}} \ll \mathrm{k}_{-\mathrm{d}}$, the process becomes reaction controlled, i.e., $\theta_{e x} \approx \mathrm{k}_{\mathrm{d}} \cdot \mathrm{k}_{\mathrm{ex}} / \mathrm{k}_{-\mathrm{d}}$, and if $\mathrm{k}_{\mathrm{ex}} \gg \mathrm{k}_{-\mathrm{d}}$, the process becomes diffusion controlled, i.e., $\theta_{\mathrm{ex}} \approx \mathrm{k}_{\mathrm{d}}$.

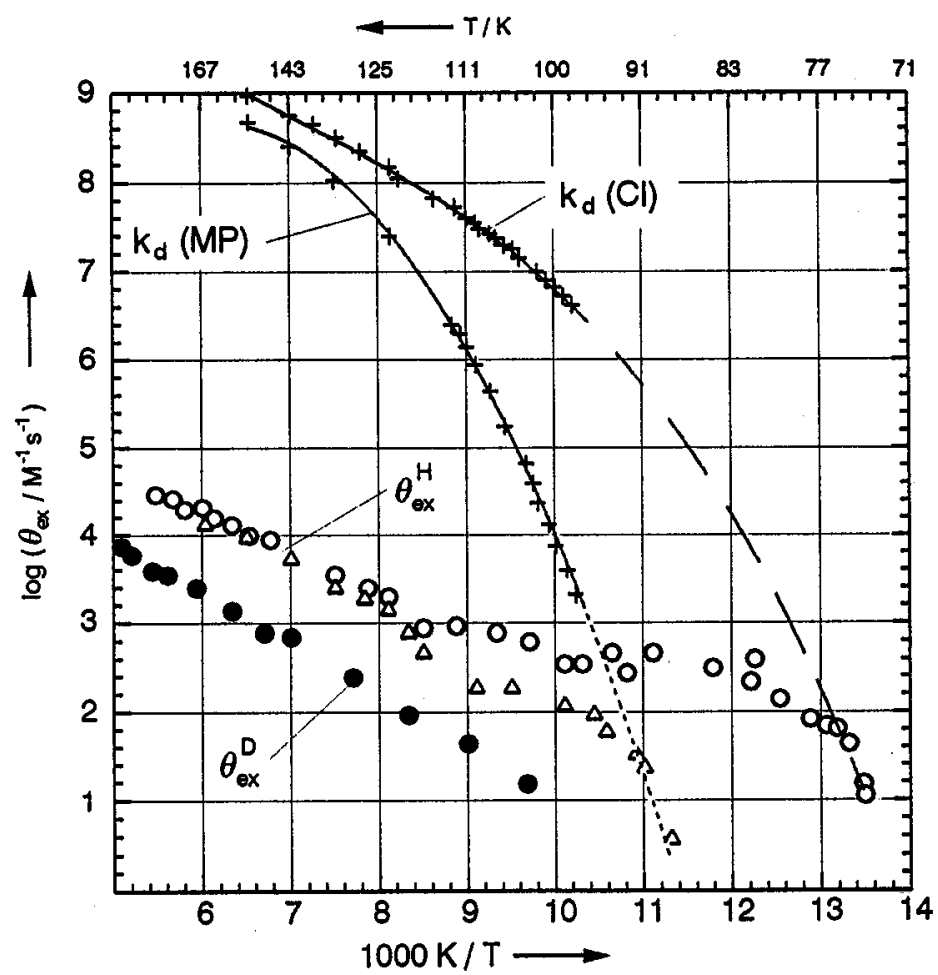

Figure 1: Arrhenius plot of experimentally determined second-order rate constants $\theta_{\text {ex }}$ in $3 \mathrm{MP}$ (triangles) and $\mathrm{CI}$ (dots and circles) and of diffusion rate constants calculated from viscosity data.

We determined $\theta_{\text {ex }}$ in the temperature range $74 \mathrm{~K}$ to $180 \mathrm{~K}$ in the two nonpolar solvents $3 \mathrm{MP}$ and the mixture cyclopentane/isopentane, $1: 4$ by volume (CI). The result is shown as Arrhenius plot in Fig. 1. For both solvents accurate viscosity data are available (ref. 4). Included in Fig. 1 are the diffusion rate constants $\mathrm{k}_{\mathrm{d}}(\mathrm{MP})$ and $\mathrm{k}_{\mathrm{d}}(\mathrm{CI})$ which were calculated using the corresponding viscosity data $\eta(\mathrm{MP})$ and $\eta(\mathrm{CI})$ and assuming $\mathrm{k}_{\mathrm{d}}=8 \mathrm{RT} / 3 \eta$.

Obviously, above $\sim 100 \mathrm{~K}$ the exchange reaction is in both solvents reaction controlled, because the calculated diffusion rate constants $k_{d}(M P)$ and $k_{d}(C I)$ are 2 to 5 orders of magnitude larger than $\theta_{\text {ex }}$. In $3 \mathrm{MP}$, which is at lower temperatures much more viscous than the mixture $\mathrm{CI}$, the Arrhenius plot of $\theta_{\text {ex }}(\mathrm{MP})$ bends down steeply below $\sim 90 \mathrm{~K}$ and the extrapolation of the $k_{\mathrm{d}}(\mathrm{MP})$ -values (dashed line in Fig. 1) clearly shows that in $3 \mathrm{MP}$ around $90 \mathrm{~K}$ the exchange rate constant 
$\theta_{\text {ex }}^{H}$ for the light amine $A_{H}$ becomes diffusion controlled. Accordingly, in the less viscous solvent $\mathrm{Cl}$ this bending is observed at a much lower temperature $(\sim 77 \mathrm{~K})$. Moreover, between $80 \mathrm{~K}$ and $100 \mathrm{~K}$ the overall rate constant $\theta_{\mathrm{ex}}^{\mathrm{H}}$ becomes temperature independent. The latter observation strongly indicates that in $\mathrm{CI}$ at least in this horizontal part of the Arrhenius plot the rate of the exchange reaction is solely determined by tunnel effects. The isotope effect which one observes if the exchanging hydrogen atoms are replaced by deuterium $\left(\theta_{\text {ex }}^{\mathrm{D}}\right.$ in Fig. 1) corroborates this conclusion. In the diffusion controlled region (below $90 \mathrm{~K}$ in $3 \mathrm{MP}$ and below $75 \mathrm{~K}$ in $\mathrm{Cl}$ ) the isotope effect should vanish because it is very unlikely that $k_{d}$ is sensitive to isotopic substitution. Unfortunately, for technical reasons it was impossible to extent the measurements with $A_{D}$ to temperatures below $\sim 100 \mathrm{~K}$ (cf. Fig. 1). Decay rates with half-lifes of several days had to be determined and $3 \mathrm{MP}$ as well as $\mathrm{CI}$ tend to crystallize if kept for several days at low temperatures. For the same reason we could not perform measurements with hydrocarbon solvents which had a higher viscosity than $3 \mathrm{MP}$.

\section{CONCLUSION}

The diphenylamine derivative $\mathbf{A}$ is a compound where tunnel effects on a light-initiated bimolecular hydrogen transfer reaction could be observed for the first time. The requirements for detecting such effects are rather stringent: Intramolecular first-order processes have to be slow enough to make competing second-order processes observable. Furthermore, the temperature dependence of the solvent viscosity has to be such that the transfer process is still reaction controlled at low temperatures. The comparison of the rate data in the two solvents employed demonstrates how important it is to meet this requirement (cf. Fig.1). We feel that the temperatureindependence of the reaction rates in $\mathrm{Cl}$ between $80 \mathrm{~K}$ and $100 \mathrm{~K}$ can only be explained in terms of quantum mechanical tunnel effects. The observed isotope effects strongly indicate that above $100 \mathrm{~K}$ thermally activated hydrogen tunneling determines the exchange rates, too.

\section{REFERENCES}

1. For a recent review, see V.A. Benderskii and V.I. Goldanskii, Intern. Rev. Phys. Chem. 11, 1 (1992)

2. K.H. Grellmann, U. Schmitt and H. Weller, Chem. Phys. Letters 88,40 (1982)

3. K.H. Grellmann, U. Schmitt and H. Weller, J. Chem. Soc. Chem. Comm. 1982, 591

4. (a) H. Lesche, D. Klemp and B. Nickel, Z. Phys. Chem. 141, 239 (1984)

(b) A.A. Ruth, B. Nickel and H. Lesche, Z. Phys. Chem. in the press (1992) 